\title{
Heavy Diboson Production at the Tevatron
}

\author{
D. S. Waters ${ }^{1}$ (for the CDF and DØ Collaborations) * \\ 1- University College London, Department of Physics \& Astronomy \\ Gower Street, London WC1E 6BT, U.K.
}

\begin{abstract}
Tremendous progress has been made in recent years in the measurement of heavy boson pair-production at the Tevatron collider. I will briefly review the current status of heavy diboson production measurements at CDF and D $\varnothing$ with special emphasis on the recently released $Z Z$ cross section measurements.
\end{abstract}

\section{Introduction}

Leading order Feynman diagrams for $W W, W Z$ and $Z Z$ production include $t$-channel quark exchange and $s$-channel vector boson exchange diagrams. Measurements of the cross-sections for these processes test QCD at NLO and, through the presence of triplegauge coupling vertices in the $s$-channel diagrams, test fundamental predictions of the EWK Standard Model. Experimentally, it is important to verify the rate of production of vector boson pairs since they are significant and often irreducible backgrounds to searches for new physics, most notably in the Higgs sector. Technically, these measurements are also informing Higgs boson search strategies at CDF and $D \varnothing$.

$W W([1,2])$ and $W Z$ production $([3,4])$ are firmly established in fully leptonic decay modes and have been used to place limits on anomalous triple-gauge couplings that are complementary to those obtained at other colliders [5, 4, 6, 7]. Interestingly, a first tentative signal has been observed in the semi-leptonic mode $W W / W Z \rightarrow l \nu j j$ by the CDF experiment [8].

\section{$2 \quad Z Z$ Cross Section Measurements}

\subsection{CDF}

$\mathrm{CDF}$ have performed searches in the modes $Z Z \rightarrow \ell \ell \ell \ell$ and $Z Z \rightarrow \ell \ell \nu \nu$ in $1.9 \mathrm{fb}^{-1}$ of data [9]. A posteriori, the 4-lepton channel has the greatest sensitivity. 2 events are observed in lepton channels with a $Z+$ jets background expectation of only $0.014_{-0.008}^{+0.010}$ and a further single event in a channel with 6 times larger background. The complete breakdown is given in table 1 and the event kinematics are compared to signal and background expectations in figure 1.

The CDF search in the $Z Z \rightarrow \ell \ell \nu \nu$ channel uses a matrix-element based likelihood method to distinguish the $Z Z$ signal from the largely irreducible $W W$ background. The final result, combining both $\ell \ell \ell \ell$ and $\ell \ell \nu \nu$ channels, is a measured cross section of $\sigma(Z Z)=$ $1.4_{-0.6}^{+0.7}$ (stat.+syst.) pb, in good agreement with the prediction $\sigma(Z Z)_{\mathrm{NLO}}=1.4 \pm 0.1 \mathrm{pb}$. The probability of the background alone fluctuating to give the observed events in both channels is $5.1 \times 10^{-6}$.

* See referenced publications for a complete set of acknowledgments. The author would specifically like to thank the Royal Society for their generous support. 


\begin{tabular}{l|c|c}
\hline Category & $\begin{array}{c}\text { Candidates without } \\
\text { a trackless electron }\end{array}$ & $\begin{array}{c}\text { Candidates with } \\
\text { a trackless electron }\end{array}$ \\
\hline$Z Z$ & $1.990 \pm 0.013 \pm 0.210$ & $0.278 \pm 0.005 \pm 0.029$ \\
$Z+$ jets & $0.014_{-0.007}^{+0.010} \pm 0.003$ & $0.082_{-0.060}^{+0.089} \pm 0.016$ \\
\hline Total & $2.004_{-0.015}^{+0.016} \pm 0.210$ & $0.360_{-0.060}^{+0.089} \pm 0.033$ \\
\hline Observed & $\mathbf{2}$ & $\mathbf{1}$ \\
\hline
\end{tabular}

Table 1: The breakdown of expected and observed numbers of events in 2 separate event classes in the CDF search for $Z Z \rightarrow \ell \ell \ell$. Note that events containing a forward electron with no matching track suffer a significantly higher background contamination. Overall sensitivity is optimised by keeping the two sub-sets separate throughout the analysis.

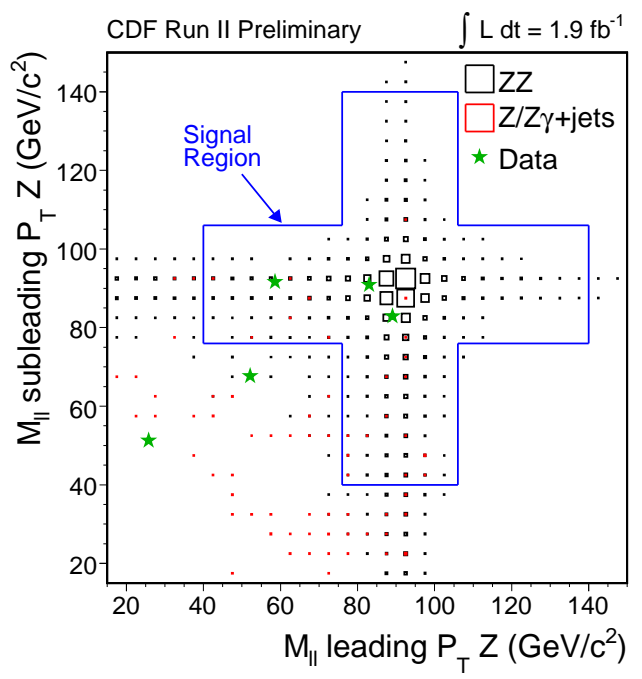

Figure 1: The distribution of event candidates in CDF's search for $Z Z \rightarrow \ell \ell \ell \ell$ events. The three stars inside the signal region are the final candidate events, compared to the expected distributions of both signal and background processes. 


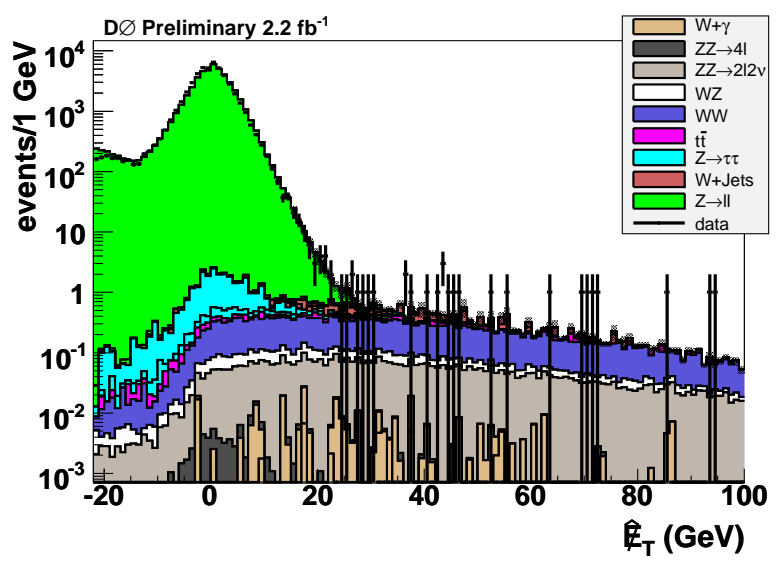

Figure 2: The distribution of effective missing- $E_{T}$ for $Z Z \rightarrow e e \nu \nu$ signal and backgrounds. A cut is made requiring this quantity to be larger then $30 \mathrm{GeV}$, effectively selecting a sample of $W W$ and $Z Z$ events that are then separated statistically.

\section{$2.2 \mathrm{D} \varnothing$}

$\mathrm{D} \varnothing$ have searched for $Z Z$ production in the 4-lepton mode in an earlier data set and used the resulting upper limit to place constraints on non-Standard Model $Z Z\left(Z / \gamma^{*}\right)$ couplings [7].

Recently, D $\varnothing$ have searched for $Z Z$ production in the mode $Z Z \rightarrow \ell \ell \nu \nu$ in $2.2 \mathrm{fb}^{-1}$ of data [10]. They construct an effective missing- $E_{T}$ object, which gives extra weight to the most reliable components of the missing- $E_{T}$ measurement. The distribution of this quantity in the di-electron channel is shown in figure 2. As can be seen, a cut can be made which selects a relatively pure sample of $W W$ and $Z Z$ events. The events are separated statistically by forming a likelihood discriminant based on the (di-)lepton kinematics. The probability for both $e e$ and $\mu \mu$ samples to be comprised entirely of non- $Z Z$ events is $8.2 \times 10^{-3}$. Equivalently, a tentative cross section can be quoted : $\sigma(Z Z)=2.1 \pm 1.2$ (stat.+syst.) pb. DØ quote a theoretical cross section for comparison of $\sigma(Z Z)_{\mathrm{NLO}}=1.6 \pm 0.1 \mathrm{pb}$. Note that the CDF quoted cross section has been computed in the zero-width approximation while the D $\varnothing$ number includes lower mass $\gamma^{*}$ contributions; however in both cases the measured cross sections have been corrected appropriately to provide a true comparison with theory.

\section{Conclusions and Outlook}

Figure 3 summarises recent cross section measurements in the electroweak sector at the Tevatron. Precision measurements are now being made of many diboson final states, and the last 2 years have seen both $W Z$ and $Z Z$ signals firmly established for the first time. So far, measured cross sections are in line with Standard Model expectations, confirming important predictions and placing further constraints on non-Standard Model couplings.

Excitingly, increased luminosity together with technical advances made partly through the measurement of heavy diboson production will soon yield experimental sensitivity to Standard Model Higgs production in certain mass ranges, as indicated in figure 3. 


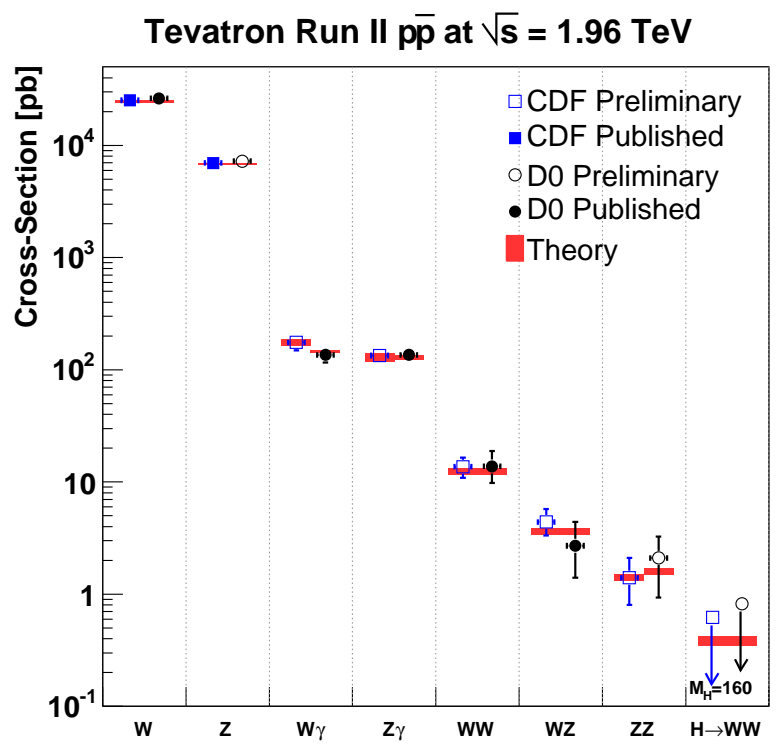

Figure 3: A summary of electroweak cross section measurements at Fermilab's Tevatron collider. The new $Z Z$ cross section measurements described here are indicated second from right.

\section{References}

[1] D. Acosta et al. (The CDF Collaboration), Phys. Rev. Lett. 94, 211801 (2005).

[2] V. M. Abazov et al. (The D $\varnothing$ Collaboration), Phys. Rev. Lett. 94, 151801 (2005).

[3] A. Abulencia et al. (The CDF Collaboration), Phys. Rev. Lett. 98, 161801 (2007).

[4] V. M. Abazov et al. (The D $\varnothing$ Collaboration), Phys. Rev. D 76, 111104(R) (2007).

[5] T. Aaltonen et al. (The CDF Collaboration), Phys. Rev. D 76, 111103 (2007).

[6] V. M. Abazov et al. (The DØ Collaboration), Phys. Rev. D 74, 057101 (2006).

[7] V. M. Abazov et al. (The DØ Collaboration), Phys. Rev. Lett. 100, 131801 (2008).

[8] CDF-Note 9216; http://www-cdf.fnal.gov/physics/ewk/2008/www/

[9] T. Aaltonen et al. (The CDF Collaboration), Phys. Rev. Lett. 100, 201801 (2008).

[10] DØ Conference Note 5620; http://www-d0.fnal.gov/Run2Physics/WWW/results/prelim/EW/E24/ 